\title{
Site-specific isotope effects and origin inference
}

\author{
M.L. Martin and G.J. Martin
}

CEAIS, Site de la Géraudière, Rue P.A. Bobierre, BP. 72304, 44323 Nantes Cedex 03, France

The isotope contents existing at natural abundance are governed by a complex set of parameters linked to both synthetic and biosynthetic mechanisms and to environmental conditions. Whereas isotope ratio mass spectrometry (IRMS) precisely measures the overall isotope content of a molecular species, NMR enables site-specific natural isotope fractionation to be studied (SNIF-NMR). From a fundamental point of view the isotopic pattern of a given molecule is a rich source of information on the reaction pathway and in particular on the genealogy of the atoms, on the relative rates of certain reaction steps, on the percentages of intra- and inter-molecular hydrogen transfer and possibly on the stereospecificity of the reactions... Consequently it is not surprising that the natural abundance isotope ratios provide powerful analytical criteria for authenticating the origin of products. Used either alone, or combined with IRMS, the SNIF-NMR method is capable of unambiguously characterizing an increasing number of products in terms of synthetic or natural origin, botanical nature of the plant precursor, geographical origin and, sometimes, year of production. Many applications in the fields of fruit juices, wines and alcoholic beverages, aromas, oils, drugs... have now been developed.

\section{Introduction}

Isotope ratio mass spectrometry (IRMS) is recognized as a sensitive and precise technique for determining isotopic contents at natural abundance. Since the fifties many raw materials, such as wood, or isolated molecular species, such as cellulose, have been investigated [1-7]. Thus the isotopic parameters of carbon, hydrogen, oxygen and nitrogen have become rich sources of information in domains as various as paleoclimatology, environment, plant metabolism, agronomy, nutrition, medicine and so on.

In IRMS the product is first burnt to give principally carbon anhydride and water. The isotope ratio of carbon is measured on $\mathrm{CO}_{2}$ whereas that of hydrogen is determined on the hydrogen gas obtained by reduction of water. Consequently the method gives access only to an overall isotope content averaged over the different molecular positions. The isotopic ratio corresponding to a given site, for instance the carbonyl or the methyl site of acetic acid, can be determined by IRMS [8] but at the price of lengthy degradations of the compound to isolate the atom under consideration. Obtaining the whole set of specific isotope contents of a given molecule by IRMS therefore requires appropriate chemical transformations in which possible fractionation effects are avoided or rigorously estimated.

In 1981 we showed that atomic discrimination due to chemical shift makes NMR a powerful instrument for investigating Site-specific Natural Isotope Fractionation (SNIFNMR), specially in the case of hydrogen [9]. This approach has now been widely exploited either alone or combined with IRMS determinations.

\section{The different types of basic information provided by site-specific isotope ratios}

Initially proposed for solving an important problem both in France and in Europe, the detection of illegal enrichment of wines (chaptalization) [10], the SNIF-NMR method now provides new or improved information in a wide range of fundamental or applied areas:

- A simultaneous determination of primary and secondary kinetic isotope effects involving the whole set of observable isotopomers of a given molecular species is possible, without the need for selective enrichment [11-16]. The evolution of the isotope ratio at a given position $i$ of reactant $A,(D /$ $H)_{i}^{A}$, as a function of the fraction of reaction, $\rho$, and of the primary, $k_{H} / k_{D}$, or secondary, $k_{H} / k_{H}^{D}$, kinetic isotope effects, is illustrated in figure 1 for a non-branched reaction. The fractionation factor can be obtained from the ratio of the values of $(D / H)_{i}^{A}$ at yield $\rho$ and at the beginning of the reaction [11]

$$
\left(\frac{k_{H}}{k_{D}}\right)^{-1}=1+\frac{\ln \left((D / H)_{i}^{A} /(D / H)_{i}^{A o}\right)}{\ln (1-\rho)}
$$

Even in the case of small secondary isotope effect, $k_{H} / k_{H}^{D}$, the variation of $(D / H)_{i}^{A}$ may be large enough, specially at high yield of the reaction (Fig. 1), to ensure a satisfactory precision for the determination $[11,16]$.

- The thermodynamic isotope effects occurring in systems at equilibrium can be determined with the same 
advantages as above. For instance hydroxylic exchanges in hydro-alcoholic mixtures, [17], or keto-enol interconversion in tautomeric equilibria [18] are isotopically characterized in a single experiment.

- All gas-liquid fractionation factors associated with the observable isotopomers (hydrogen, carbon or oxygen...) of a given molecule are accessible in the same set of distillation experiments [19].

- A matrix, [A], of isotopic coefficients, $a_{i j}$, connecting molecular sites $i$ of reactants to sites $j$ of products in chemical or biochemical reactions of any level of complexity can be defined [20]

$$
[\mathrm{A}] \overrightarrow{\mathbf{D}^{\mathbf{S}}}=\overrightarrow{\mathbf{D}^{\mathbf{Q}}}
$$

where $\overrightarrow{\mathbf{D}^{\mathbf{S}}}$ and $\overrightarrow{\mathbf{D}^{\mathbf{Q}}}$ are the column vectors of the site-specific isotope ratios of the starting molecules and end products respectively.

For instance, the isotopic transfer coefficients connecting sites $j$ of the glycolytic products, ethanol, water, and glycerol, to sites $i$ of the starting materials, glucose and water, in a fermentation reaction, are rich sources of information on the genealogy of the different hydrogens and consequently on the mechanistic pathway. In particular the $a_{i j}$ values provide information

- on the stereospecificity of the reduction step of dihydroxyacetone phosphate,

- on the relative contributions of the intra- and inter-molecular transfers of hydrogen 2 of glucose by phosphoglucose isomerase,

- on the relative rates of certain reaction steps etc. The influence of the reaction conditions (temperature, nature of the microorganism, composition of the medium...) on these coefficients enables possible modifications of the reaction mechanism to be detected and interpreted [21].

From a general point of view the natural abundance site-specific isotope ratios of a given product (ethanol for instance) are useful criteria for inferring many properties of the precursors (sugars), even when these precursors are fully exhausted or inaccessible.

\section{The site-specific isotope ratios determined by NMR}

The isotopic content at a specific molecular position $i$ may be described in terms of a natural abundance parameter $A_{i}$

$$
A_{i}=\frac{\left[H_{i}\right]}{\left[H_{i}\right]+\left[L_{i}\right]}
$$

where $\left[H_{i}\right]$ and $\left[L_{i}\right]$ are respectively the numbers of heavy and light isotopes at site $i$.

It may also be characterized by the isotope ratio $R_{i}$

$$
R_{i}=\frac{\left[H_{i}\right]}{\left[L_{i}\right]} .
$$

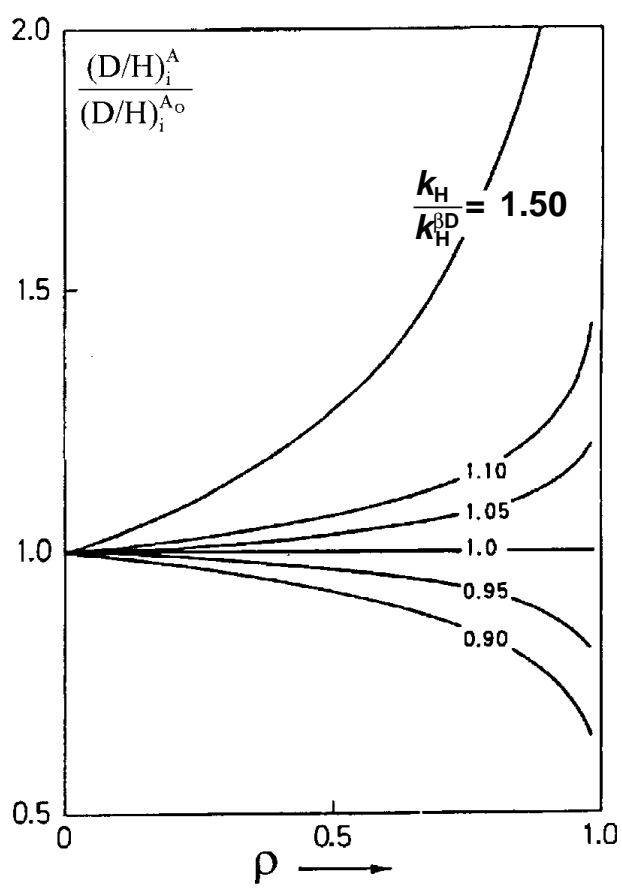

Figure 1. Evolution of the isotope ratio at position $i$ (adjacent to the reacting site) of reactant $\boldsymbol{A}$ involved in a non-branched reaction, as a function of the yield, $\rho$, and of the secondary isotope effect $k_{\mathrm{H}} / k_{\mathrm{H}}^{\mathrm{BD}}$ [11]. Relatively large variations in the deuterium content of site $i,(D / H)_{i}^{A}$, may occur at low residual concentrations of $A$, even for values of $k_{H} / k_{H}^{B D}$ close to unity.

This ratio is of the order of $1.510^{-4}$ for hydrogen and $1.110^{-2}$ for carbon for instance.

Alternatively a site-specific relative deviation, $\delta_{i}$, may be defined as

$$
\delta_{i}(\% o)=\frac{R_{i}-R_{\text {ref }}}{R_{\text {ref }}} 1000
$$

where $R_{\text {ref }}$ is the isotope ratio of an international standard $[22,23]$ which is a carbonate (PDB) for carbon and mean ocean water (V.SMOW) for hydrogen.

At the high isotopic dilution of hydrogen the value of the isotopic abundance, $A_{i}$, is nearly equal to that of the isotope ratio, $R_{i}$, but the difference between $A_{i}$ and $R_{i}$ is no longer negligible in the case of carbon.

In NMR the signal areas measured in quantitative experimental conditions are simply proportional to the numbers of nuclei involved in the resonance signals. Consequently, as illustrated by the example of nicotine extracted from tobacco leaves, the mole fractions of the monodeuterated isotopomers can be directly computed from the ${ }^{2} \mathrm{H}-\mathrm{NMR}$ spectrum (Fig. 2). Taking as reference site 3 of the pyridine ring, the relative isotope ratios determined for positions 1 to 12 (order of decreasing chemical shift) have the following values for a sample from Thailand: 
0.965/0.848/1/0.574/0.847/0.513/0.468/1.056/0.819/1.019/ $1.087 / 1.100$ [24]. This behaviour illustrates the existence of very strong deviations with respect to a statistical distribution of ${ }^{2} \mathrm{H}$ which would lead to values uniformly equal to unity.

In the absence of an isotopic standard, the NMR spectrum gives direct access only to the relative distribution of the isotopes in the different sites of the molecule. By adding to the sample a known amount of an appropriate reference substance, the isotope ratio of which has been previously calibrated (for instance tetramethylurea supplied by IRMM at Brussels), it is possible to determine the isotopic parameters $A_{i}, R_{i}$ or $\delta_{i}$ by using NMR spectroscopy alone. Alternatively the absolute isotope content can be computed from both the mole fractions $f_{i}$ obtained by NMR and the overall isotopic abundance of the molecule, $\bar{A}$, measured by IRMS

$$
A_{i}=\frac{f_{i}}{F_{i}} \bar{A}
$$

where $F_{i}$ are the statistical fractions.

In spite of its low natural abundance (of the order of 1.5 $\left.10^{-4}\right)$ and low intrinsic sensitivity $\left(\simeq 10^{-2}\right.$ with respect to $\left.{ }^{1} \mathrm{H}\right)$ deuterium is well suited to quantitative comparisons by NMR. Although more difficulties are encountered in the case of carbon, typical isotopic distributions can now be characterized with a reasonable level of confidence [25]. Thus it has been checked in the case of glycerol that the carbon isotope pattern determined by NMR is in good agreement with site-specific IRMS data obtained by degrading the sample into hexamethylene tetramine (carbons 1, 3) and formate (carbon 2) [26].

\section{The site-specific isotopic genealogy}

In natural products the isotope contents at the different sites of a molecule are governed by the entire set of kinetic or thermodynamic fractionation effects resulting from physicochemical transformations (evapotranspiration in the leaves) or metabolic evolutions. These isotope fractionation effects depend on the biochemical mechanism and on the yields of the different reaction steps. For a given bioconversion, whatever its complexity, the isotopic coefficients $a_{j i}$ connecting sites $j$ in the products, $Q$, to sites $i$ in the reactants, $S$, (Eq. 2) can be estimated from the isotope ratios $(D / H)_{i}^{S}$ and $(D / H)_{j}^{Q}$ measured in a series of reactions conducted, in strictly identical conditions, with reactants characterized by different isotopic distributions. Thus in the case of glycerol obtained in fermentation reactions (Fig. 3) it is shown that the coefficients $a_{\mathrm{A} 6}, a_{\mathrm{B} 6}, a_{\mathrm{B} 1}, a_{\mathrm{A} 2}$ connecting respectively sites 66' 1 and 2 of glucose to sites $A\left(1_{\mathrm{S}}, 3_{\mathrm{R}}\right)$ and $B\left(1_{\mathrm{R}}, 3_{\mathrm{S}}\right)$ of glycerol (Fig. 3) exhibit relatively high values [27]. In particular the values of $a_{6 \mathrm{~A}}$ and $a_{6 \mathrm{~B}}$ are not far from the theoretical value 0.25 which would represent a fully intramolecular transfer from the methylenic hydrogens at positions 66' of glucose to the fourfold degenerated signals $A$ and $B$ of glycerol in conditions of complete conversion or negligible isotope effects. This behaviour characterizes a high degree of connectivity between hydrogens at positions

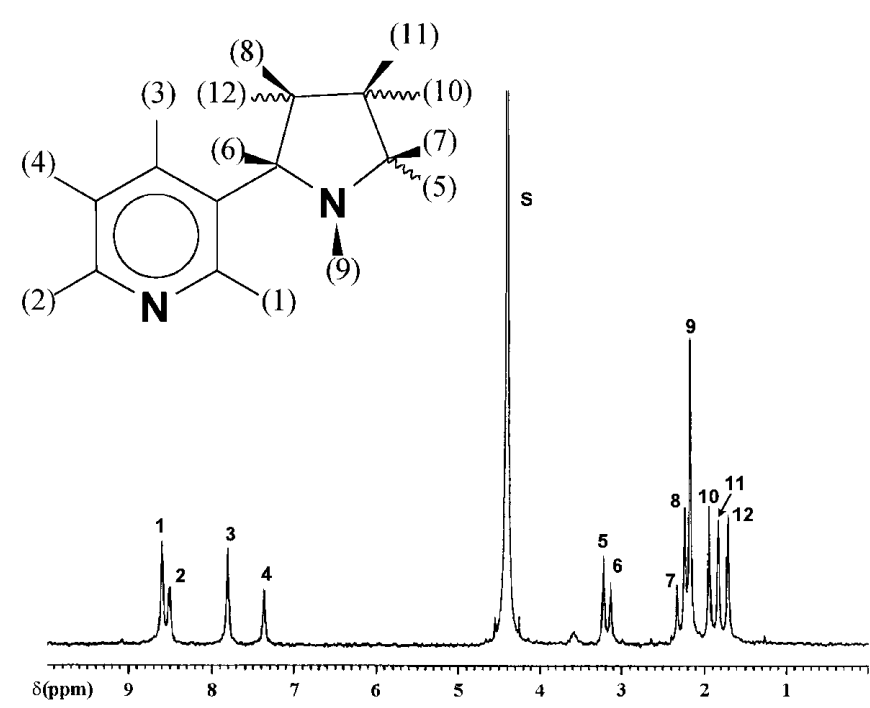

Figure 2. Natural abundance ${ }^{2} \mathrm{H}-\mathrm{NMR}$ spectrum (76.7 $\mathrm{MHz}$ ) of nicotine [24]. The solvent, $\mathrm{S}$, is $\mathrm{CH}_{3} \mathrm{NO}_{2}$.

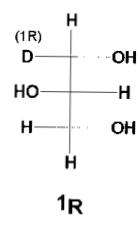

$(1 R, 2 S)$

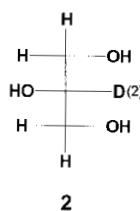

2

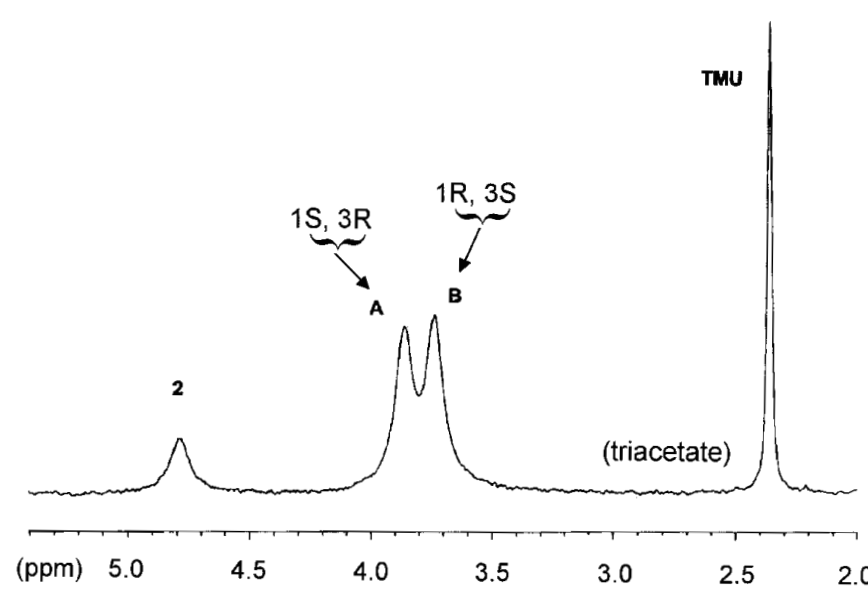

Figure 3. ${ }^{2} \mathrm{H}-\mathrm{NMR}$ spectrum $(76.8 \mathrm{MHz})$ of the triacetate of a glycerol sample obtained by fermentation of maize glucose [27]. In spite of the symmetry degeneracy five isotopomers are characterized (stereospecific numbering) since the origins of the hydrogen atoms on the two enantiotopic moieties (1S and 3R for instance) of glycerol are not the same. TMU identifies the signal of the isotopic reference, tetramethylurea. 
$1_{\mathrm{S}}$

$3_{\mathrm{R}}\left(1_{\mathrm{R}}\right.$ and $\left.3_{\mathrm{S}}\right)$ of glycerol and hydrogens at positions 2 and 6pro-R (1 and 6pro-S) of glucose. Consequently the isotopic pattern of glycerol is expected to provide a probe of the isotopic properties of its sugar precursors. As compared to ethanol, the widely exploited probe for sugars [10], glycerol exhibits similar but not fully identical connectivities. Its isotopic pattern may therefore provide some complementary information.

This genealogical approach is specially efficient to estimate the relative contribution of hydrogen transfer from water to specific positions in the products. It is obvious that when the percentage of intermolecular transfer to a given site $j$ is very high, the site-specific isotope ratio $(D / H)_{j}$ no longer characterizes properties of the plant carbohydrates but reflects the behaviour of the aqueous medium. In this case $(D / H)_{j}$ is likely to have stored information on the evapotranspiration processes undergone by the plant precursor.

\section{Origin inference and authenticity criteria}

Since, as discussed above, the isotopic pattern of a molecule is governed by the mechanistic pathway and the environmental conditions of the synthesis it may be very helpful for answering a number of questions concerning the origin of the product. Thus the natural or synthetic origin of the molecule is usually very easily identified. Moreover, for natural products, the isotopic fingerprint is frequently typical of the metabolic pathway of the plant precursor $\left(\mathrm{C}_{3}, \mathrm{C}_{4}, \mathrm{CAM}\right.$...). For a given metabolic origin the isotopic distribution may enable the botanical species to be identified and for a given plant species the geographical origin may often be ascertained [11]. Thus, ethanol and water obtained in standardized fermentations of sugars can be used as a common isotopic probe for characterizing these sugars whatever their molecular structure (monosaccharides, disaccharides, polymeric structures). Figure 4 illustrates the hierarchical clustering ability of the hydrogen and carbon isotopic parameters to infer the botanical nature of the plant precursor [28]. In certain cases it is even possible, for a given molecule extracted from a given plant species grown in a given region, to get information on the year of harvest of the plant.

\section{Conclusion}

Exploited alone, or more often in combination with overall carbon isotope ratios measured by IRMS (see other articles in this document), the SNIF-NMR fingerprint has already greatly contributed to establishing the notion of authenticity of food and beverages on well defined quantitative criteria. The method has now been adopted, and is currently used, by official regulating organisms in Europe and the United States. Further examples, in particular in the field of aroma components, can be found in references 29 to 38. Many IRMS studies in other domains such as agronomy [6], nutrition, medicine [39-40], will certainly benefit from SNIF-

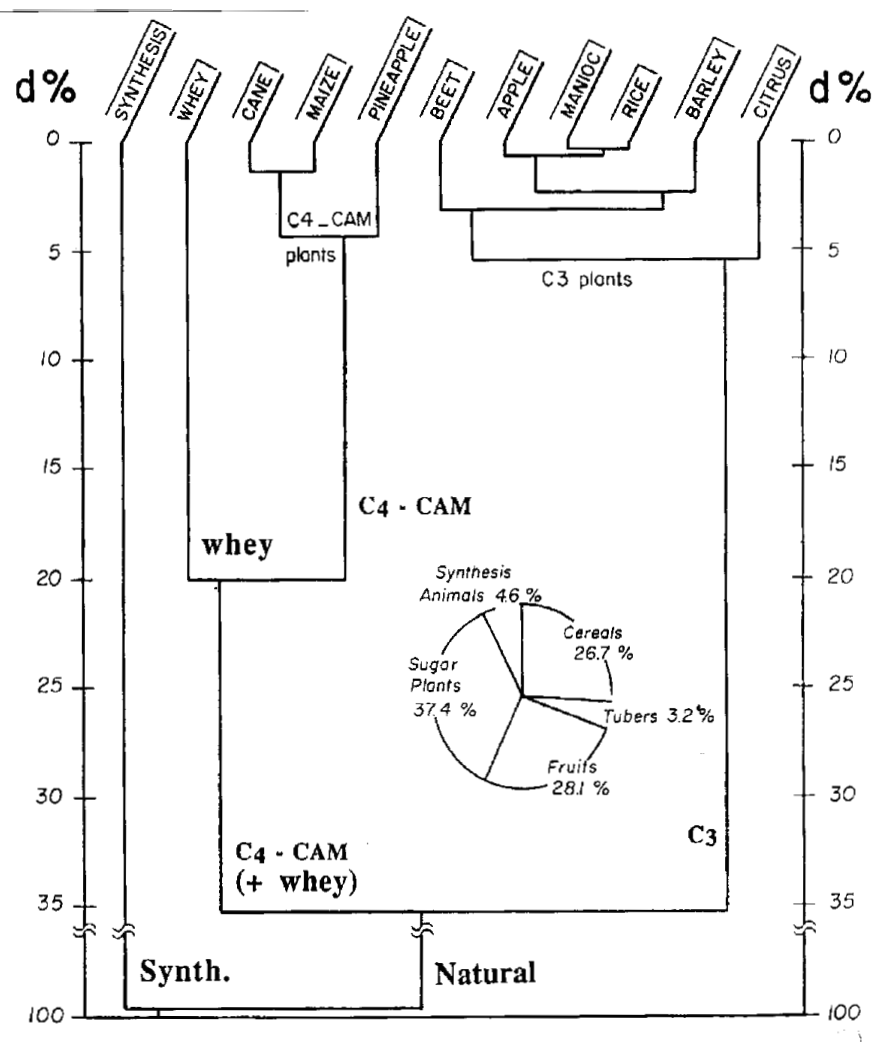

Figure 4. Hierarchical clustering of ethanols obtained by fermentation of sugars from different botanical origins [28]. The classification is built on the euclidian similarity between groups characterized by the site-specific hydrogen isotope ratios of ethanol, the overall ${ }^{13} \mathrm{C}$ content of ethanol and the hydrogen isotope ratio of the fermentation medium. The sample distribution used in the analysis is given in a circle.

NMR development when appropriate isotopic probes become more firmly established.

\section{References}

1. Craig, H. J. Geol. 1954, 62(2), 115-149.

2. Park, R.; Epstein, S. Geochim. Cosmochim. Acta 1960, 21, 110-126.

3. Schiegl, W. E.; Vogel, J. C. Earth Planet. Sci. Lett. 1970, 7, 307-313.

4. Smith, B. W.; Epstein, S. Plant Physiol. 1971, 47, 380-384.

5. Sternberg, O. L. Q.; Deniro, M. J.; Ting, I. P. Plant Physiol. 1984, 74, 104-107.

6. Francey, R. J.; Farquhar, G. D. Nat 1982, 297, 28-31.

7. Epstein, S.; Yapp C. J.; Hall, J. H. Earth Planet. Sci. Lett. 1976, 30, 241-251.

8. Meinschein, W. G.; Rinaldi, G. G. L.; Hayes, J. M.; Schoeller, D. A. Biomed. Mass Spectom. 1974, 1, 172-174.

9. Martin, G. J.; Martin, M. L. Tetrahedron Lett. 1981, 22, 35253528. 
10. Martin, M. L.; Martin, G. J. In Modern Methods of Plant Analysis; Linskens, H. F. and Jackson, J. F. Eds.; Springer Verlag, Berlin 1988; Vol. 6, pp 258-275.

11. Martin, M. L.; Martin, G. J. In NMR Basic Principles and Progress; Diehl, P.; Fluck, E.; Günther, H.; Koofeld, R. and Seelig, J. Eds.; Springer Verlag, Berlin 1988; Vol. 23, pp 1-69.

12. Pascal, R. A.; Baum, M. W.; Wagner, C. K.; Rodgers, L. R. J. Am. Chem. Soc. 1984, 106, 5377-5378.

13. Leopold, M. F.; Epstein, W. W.; Grant, D. M.; J. Am. Chem. Soc. 1988, 110, 616-617.

14. Zhang, B. L. Magn. Res. Chem. 1988, 26, 955-959.

15. De Rosa, M.; Shadle R. G.; Foster, N. J. CS Chem. Comm. 1993, 1757-1758.

16. Singleton, D. A.; Thomas, A. A. J. Am. Chem. Soc. 1995, 117, 9357-9358.

17. Zhang, B. L.; Martin, M. L. J. Am. Chem. Soc. 1992, 114(18), 7089-7092.

18. Zhang, B. L.; Mabon, F.; Martin, M. L. J. Phys. Org. Chem. 1993, 6, 367-373.

19. Moussa, I.; Naulet, N.; Martin, M. L.; Martin, G. J. J. Phys. Chem. 1990, 94, 8303-8309.

20. Martin, G. J.; Zhang, B. L.; Naulet, N.; Martin, M. L. J. Am. Chem. Soc. 1986, 108, 5116-5122.

21. Zhang, B. L.; Yunianta; Vallet, C.; Martin, Y. L.; Martin, M. L. Bioorg. Chem. 1997, 25, 117-129.

22. Hagemann, R.; Nief, G.; Roth, E. Tellus 1970, 22(6), 712-715.

23. Craig, H. Geochim. Cosmochim. Acta 1957, 12, 133-149.

24. Jamin, E.; Naulet, N.; Martin, G. J. Plant Cell Evironm. 1997, 20, 589-599.

25. Zhang, B. L.; Buddrus, S.; Trierweiler, M.; Martin, G. J. J. Agric. Food Chem. 1998, 46, 1374-1380.
26. Zhang, B. L.; Trierweiler, M.; Jouitteau, C.; Martin, G. J. Anal. Chem. 1999.

27. Zhang, B. L.; Buddrus, S.; Martin, M. L. 1999, Bioorg. chem.

28. Martin, G. J.; Danho, D.; Vallet, C. J. Sci. Food Agric. 1991, 56, 419-434.

29. Grant, D. M.; Curtis, J.; Croasmun, W. R.; Dalling, D. K.; Wehrli, F. W.; Wehrli, S. J. Am. Chem. Soc. 1982, 104, 44924494.

30. Toulemonde, B.; Horman, I.; Egli, H.; Derbesy, M. Helv. Chim. Acta 1983, 66(231), 2342-2345.

31. Hagedorn, M. L. J. Agric. Food Chem. 1992, 40, 634-637.

32. Hanneguelle, S.; Thibault, J. N.; Naulet, N.; Martin, G. J. J. Agric. Food Chem. 1992, 40(1), 81-87.

33. Carle; R.; Beyer, J.; Cheminat, A.; Krempp, E. Phytochem. 1992, 31, 171-174.

34. Fronza, G.; Fuganti, C.; Grasselli, P.; Barbeni, M.; Cisero, M. J. Agric. Food Chem. 1993, 41, 235-239.

35. Mosandl, A. Food Rev. Int. 1995, 11(4), 597-664.

36. Fronza, G.; Fuganti, C.; Grasselli, P.; Servi, S.; Zucchi, G.; Barbeni, M.; Cisero, M. J. Agric. Food Chem. 1995, 43, 439443.

37. Remaud, G. S.; Martin, Y. L.; Martin, G. G.; Martin, G. J. J. Agric. Food Chem. 1997, 45, 859-866.

38. Barbeni, M.; Cisero, M.; Fuganti, C. J. Agric. Food Chem. 1997, 45, 237-241.

39. Brazier, J. L. In: Les isotopes stables. Goldstein, S.; Louvet, P. and Soulié, E., Eds.; Symposium, Paris-CEA 1993; pp 310.

40. Koziet, J.; Gross, P.; Debry, G.; Royer, M. J. Biological Mass Spectrom. 1991, 20, 777-782. 